\title{
Los prácticos de laboratorio: una mirada interpretativa en prácticas de enseñanza de química en Bachillerato Diversificado*
}

I sabel Duglio*

\section{Resumen}

Este artículo presenta una investigación sobre actividades de laboratorio, en prácticas de enseñanza de química en Bachillerato Diversificado. En este plan de estudios se concibe un curso práctico que se desarrolla en paralelo con un curso teórico. Compete al docente buscar estrategias que le permitan dirimir la dualidad teoría-práctica, que surge de los lineamientos curriculares.

Se realiza un abordaje cualitativo del tema, en el cual se ha intentado focalizar la atención desde la reconstrucción de conocimiento y las interacciones que se observan en los prácticos de laboratorio.

Con relación a la reconstrucción de conocimiento se propone: la resignificación de conocimiento cotidiano, la reformulación del error y la búsqueda de integración conceptual. Desde las interacciones, se plantea: el segmento de interacción docente - alumnos, el segmento de interacción alumnos - docente, y una interacción mediada: la puesta en escena.

\begin{abstract}
This article presents an investigation on laboratory activities, in the practices of teaching Chemistry in a Diversified Baccalaureate. In this curriculum we conceived a practical course which is developed in parallel with a theoretical course.

It is the professor's competence to find strategies that will allow to dissolve the theory-practice duality, that arises from the curricular outlines.

A qualitative focus of the theme is shown, where we tried to focus the attention from the reconstruction of knowledge to the interaction observed in the laboratory practices.

With relation to the reconstruction of knowledge we propose: the resignation of daily knowledge, the reformulation of the mistake and the search for conceptual integration. From the interactions, we consider: the segment of professor-student interaction, the segment of student-professor interaction and a mediated interaction: setting the scene.
\end{abstract}

\section{I ntroducción}

La presente investigación refiere a un estudio sobre prácticos de laboratorio, en prácticas de enseñanza de química en Bachillerato Diversificado ${ }^{1}$, en un centro educativo de la ciudad de Rivera (Uruguay). En el plan de estudios mencionado, se concibe un "curso práctico" que se desarrolla en paralelo con un "curso teórico".

Este trabajo surge del interés y necesidad sentida de investigar los prácticos de laboratorio, buscando entender qué aportan a la enseñanza de la química y cuáles son los soportes teóricos que los tornan significativos para las buenas prácticas de enseñanza. 
Cuando se menciona "buenas prácticas de enseñanza" no representa un hecho menor en nuestra investigación, debido a que la muestra se constituye con docentes que son reconocidos como ejemplos de buenas prácticas en el medio donde se desempeñan.

Al referirnos a buena enseñanza, queremos ubicarnos en lo que propone Fenstermarcher (1989), la palabra "buena" utilizada como adjetivo para la enseñanza tiene tanto fuerza moral como epistemológica. Buena enseñanza en el sentido moral, representa un accionar docente capaz de generar acciones de principios en los alumnos. Buena enseñanza en el sentido epistemológico, representa una enseñanza racionalmente justificable, digna y válida de ser conocida por los alumnos.

Es importante considerar que la conceptualización de buena enseñanza expresada anteriormente, no es ajena a las identidades propias de la comunidad educativa en cuestión, así como tampoco de la disciplina, desde donde miramos las prácticas de enseñanza. Como menciona Stodolsky (1991), lo que se enseña condiciona profundamente la actividad del docente. En nuestro recorrido por las aulas, si bien todos los docentes trabajan con las mismas guías de laboratorio ${ }^{2}$, encontramos que no hay uniformidad y que las actividades no se homogeinizan porque lo que cuenta es el estilo de enseñanza, del docente que interactúa con un grupo en un contexto determinado.

Los propósitos pasaron por observar prácticas de enseñanza de química, en búsqueda de regularidades y particularidades en los prácticos de laboratorio; por analizar las observaciones y buscar analogías con procesos que caracterizan a la actividad científica, así como también, interpretar la dinámica de la implementación de los prácticos de laboratorio y la percepción de los actores involucrados, para construir desde los hallazgos nuevos aportes que resulten significativos en la realidad estudiada.

A continuación se desarrolla una síntesis del trabajo de investigación, comenzando por los elementos teóricos que la enmarcan: las decisiones metodológicas, el camino recorrido en el procesamiento de la evidencia empírica, las dimensiones utilizadas en el análisis, los hallazgos y por último un espacio abierto a la reflexión sobre los logros alcanzados, los pendientes que quedaron por el camino y los nuevos cuestionamientos que surgieron durante el proceso.

\section{Líneas que orientan la investigación}

Con la intención de enmarcar nuestra investigación en el campo educativo, surgen como nexos referenciales el currículo y la didáctica. Ambos referenciales se cruzan y articulan cuando se quiere expresar una concepción educativa y cuando se quiere teorizar sobre las prácticas de enseñanza. Sin embargo, el currículo y la didáctica tienen perspectivas diferentes: el currículo surge históricamente para atender los procesos institucionales, y la didáctica para abordar los problemas de la enseñanza ${ }^{3}$. El pensamiento curricular forma parte del pensamiento social, lo cual enfatiza la complejidad que se encuentra en el momento de su definición; surgen significados diversos según grupos sociales y corrientes de pensamiento diferentes. No obstante podemos encontrar algunas líneas directrices en el desarrollo de los temas curriculares: un proyecto sobre lo que se pretende en el ámbito educativo, cómo se organiza en la institución educativa y cómo se proyecta en las prácticas de enseñanza.

La concreción de un currículo atiende a una forma de entender la realidad, en un determinado contexto histórico y social en el que se ubica la cultura y las instituciones educativas, y por lo tanto es necesario analizar los intereses, opciones y perspectivas que lo orientan (Gimeno et al,1995).

\section{2 - Universidad ORT Uruguay}


En las instituciones educativas y especialmente dentro del aula, ocurren procesos de socialización, y los contenidos del currículo real son los que participan de esa socialización.

En una nueva perspectiva curricular, las prácticas de enseñanza se perciben complejas ya que no se deja de tener presente la lógica disciplinar en el abordaje de los contenidos, ni el ensamblaje de los mismos con los esquemas cognitivos del alumno, sino que todo esto se integra a la intencionalidad del docente que teoriza sobre sus prácticas de enseñanza.

La didáctica de las ciencias naturales en los últimos años ha sufrido un importante proceso clarificador. Ha pasado, de ser un conjunto de prescripciones curriculares de carácter tecnicista que pretendía trasladar a la escuela una lógica positivista de la ciencia y de su metodología, a ser una disciplina posible, que dispone de los requisitos indispensables para ser considerada como tal, según Bunge (1976): una ubicación epistemológica más clara; una comunidad investigadora incipiente; un cuerpo de conocimientos específicos; un objeto de estudio; una problemática reconocida por dicha comunidad y unos medios de comunicación y contraste (revistas, congresos, etc). La didáctica de las ciencias de la naturaleza es una disciplina emergente, y tiene una construcción histórica de existencia formal muy breve. En el campo disciplinar, hace parte de muchas crisis teóricas, por sus múltiples e imprescindibles relaciones con otras disciplinas (Camilloni, 1997).

Hoy podemos concebir la didáctica de las ciencias de la naturaleza, como una disciplina autónoma, centrada en la enseñanza de los contenidos de las ciencias y enriquecida por los avances de otras disciplinas. Esto no supone que la teorización sobre la enseñanza de una ciencia derive de la propia ciencia erudita, sino que la didáctica de las ciencias de la naturaleza representa un campo disciplinar autónomo (Adúriz Bravo,2000e). Como dice Pozo (1996), lo psicológico y lo didáctico son divergentes por su objeto y campo de estudio; es decir, no por constituirse lo cognitivo en un aspecto central del campo didáctico, lo didáctico pasa a ser psicológico.

El modelo tecnicista de concebir la práctica ha logrado amplia difusión en el medio educativo, y de allí que se entienda la práctica como teoría aplicada. Es aquí donde surge la necesidad de rescatar la relación teoría - práctica, haciendo hincapié en que toda práctica tiene un referente teórico. Este referente teórico puede estar explicitado e identificado con diferentes líneas del pensamiento, o hacer parte de las creencias del docente, conformadas por las teorías implícitas, de las cuales no siempre se tiene conocimiento. En realidad, en toda práctica el referente teórico tiene un poco de cada uno de estos componentes, lo explícito y lo implícito, pero también tiene mucho de intuitivo. La relación teoría - práctica, según Cañal (1987), podemos entenderla en términos de pensamiento y acción, con un enfoque individualista, que presupone una visión unilateral y racionalista, según la cual, las ideas orientan y dirigen la práctica. Pero también, en contraposición, tenemos a quienes consideran la relación teoría - práctica como un proceso público, expresado en términos de relaciones y estructuras sociales, es decir, como un juego de roles. Este perfil de roles se torna complejo cuando se cruzan la teoría y la práctica o viceversa. Las prácticas cobran significado cuando se teoriza sobre ellas, y las teorías adquieren significado histórico, social y material en la práctica.

Cuando se interroga a los alumnos sobre cuáles son las actividades que para ellos resultan más atractivas en las clases de química, la respuesta que surge como una constante es: la propuesta de actividades de laboratorio. Suelen contraponer esta opción de una enseñanza experimental con una enseñanza libresca, que resulta poco interesante y desmotivadora. Se puede afirmar que este parecer de los alumnos es compartido por la mayoría de los docentes, si bien las diversas situaciones que enfrentan a diario: falta de instalaciones y de material 
adecuado, laboratorios que se utilizan como salones de clase, un excesivo número de alumnos, etc. no les permite realizar todas las actividades prácticas posibles. Esta percepción que exalta el papel de la experimentación en la enseñanza de las ciencias de la naturaleza, oculta una concepción ingenua que busca la posibilidad de realización de muchos prácticos de laboratorio, la respuesta a los inconvenientes que plantea el aprendizaje de las ciencias de la naturaleza. El papel que juega la experimentación en las ciencias de la naturaleza ha sido siempre sobrevalorado; ejemplo de esto lo tenemos en que son llamadas generalmente ciencias experimentales. Es pertinente precisar que la experimentación, si bien ocupa un lugar importante dentro del conocimiento científico, no representa la base del mismo, lo que realmente soporta el conocimiento son las teorías.

Si bien las teorías contienen en sí mismas la realidad, lo interpretado sobre lo real, la experimentación es una forma de contrastar esa realidad y es la que pone en evidencia la imbricada relación entre el conocimiento y el hacer, entre el pensamiento y la acción.

En la literatura y en las diferentes actividades académicas (congresos, simposios), que refieren a didáctica de las ciencias de la naturaleza, aparecen con frecuencia mencionados los prácticos de laboratorio. La mayoría de las propuestas hacen referencia a cómo renovar los prácticos de laboratorio para nivel de enseñanza media, con la mirada orientada a facilitar el proceso de aprendizaje de los alumnos.

Varios autores, Corominas (1994), Lozano (1994), Grau (1994), Caamaño (2003) mencionan los prácticos de laboratorio, como las actividades más representativas de la enseñanza de las ciencias de la naturaleza por permitir una multiplicidad de objetivos: la familiarización, observación e interpretación de los fenómenos, el contraste de hipótesis en los procesos de modelización y conceptualización en diferentes niveles de abstracción; adquisición de habilidades prácticas; aplicación de estrategias de investigación en la resolución de problemas teóricos y prácticos. Así también, se encuentran diferentes clasificaciones de prácticos de laboratorio, en función de sus objetivos: experiencias, experimentos ilustrativos, ejercicios prácticos e investigaciones (Woolnough y Allsop,1985, en: Insaustí, 1997). Toda la bibliografía indica una tendencia a la realización de actividades del tipo resolución de problemas, de corte investigativo, donde es posible alcanzar diferentes niveles de investigación.

Según plantea Caamaño (2003), los prácticos de laboratorio se realizan por las siguientes razones: motivan al alumno, permiten vivenciar los fenómenos e interpretarlos mediante variables observables, ayudan en la comprensión de conceptos, proporcionan experiencia en el manejo de instrumentos, permiten acercarse a la metodología y los procedimientos propios de la actividad científica y constituyen una opción para el trabajo en equipo.

Por otro lado, Carmen (2000), señala que las dimensiones que atraviesan los prácticos de laboratorio y permiten evaluarlos son: la dimensión social y las posibles interacciones sociales que se establecen a partir de la actividad; los conocimientos previos necesarios para realizar el trabajo práctico; cómo se relacionan con la teoría; nivel de complejidad del diseño experimental; manejo de los tiempos; análisis de resultados.

Más allá de las propuestas constructivistas, que entienden los prácticos de laboratorio como una herramienta viable en la construcción del conocimiento, cuando el punto de partida es un problema sentido por el alumno, consideramos que es importante destacar que toda actividad cobra significado acorde al nivel de discusión y análisis que se plantea en torno de ella. 
Es necesario precisar que la riqueza de una actividad experimental no pasa por la complejidad del diseño, ni por los resultados que se puedan obtener en la búsqueda de respuestas, sino que lo realmente es importante para el desenvolvimiento de estrategias cognitivas por parte del alumno, son los niveles del lenguaje que utiliza para comunicar, discutir y argumentar sus resultados, así como también el nivel de análisis que realiza, desde la observación de detalles pequeños hasta proyectarse en situaciones hipotéticas de aplicación y resolución.

En la enseñanza de la química, los prácticos de laboratorio suelen ocupar un lugar destacado cuando se intenta potenciar en los alumnos, la idea de un cuerpo de conocimiento integrado, dinámico y relevante para explicar la realidad. Sin embargo, esto no siempre sucede así, ya que suelen adquirir ideas aisladas, sin conexión con la vida cotidiana (Linn y Songer, citado en: Rodríguez Moneo, 1999).

Las actividades prácticas, más allá de los lineamientos teóricos, que sustente la intencionalidad del docente al realizarlas, constituyen unidades con significado en la secuencia didáctica que las incluye.

Al definir qué entendemos por "prácticos de laboratorio", es pertinente precisar que también podemos referirnos a ellos, como trabajos prácticos o actividades prácticas. Cuando decimos prácticos de laboratorio, hacemos referencia a actividades que promueven las relaciones interpersonales de todos los involucrados, alumnos con alumnos, alumnos con docentes, en una acción participativa, mediada por recursos materiales.

La acción emprendida en un práctico de laboratorio, bajo diferentes intencionalidades didácticas, tiene que potenciar la integración participativa, es decir, los alumnos tienen que vivenciar, los nexos que la propuesta de enseñanza plantea con el fenómeno en cuestión, tendiente a favorecer su representación o resignificación contextualizada.

\section{Abordaje metodológico para el estudio de los prácticos de laboratorio}

Al investigar los prácticos de laboratorio, surge el nexo con el aula, la inclusión de esta en una institución y más allá con el medio sociocultural, económico y político donde se insertan e interrelacionan todos los actores, fenómenos y hechos que serán objeto de estudio. Es necesario explicitar la complejidad de la situación de partida, por hacer parte de ella, y explicitar que en todo momento se buscó potenciar la objetividad construida desde el conocimiento de la propia subjetividad.

El trabajo de investigación, se realizó siguiendo un abordaje cualitativo, de carácter descriptivo e interpretativo. Al ser la investigación cualitativa esencialmente descriptiva y analítica, permitió abordar la compleja realidad de las prácticas de enseñanza, en su contexto natural, haciendo hincapié en las perspectivas de los participantes. El énfasis en el proceso investigativo se encontró en las palabras de los participantes y en su disposición a colaborar.

Los prácticos de laboratorio de química se estudian en segundo y tercer año del Bachillerato Diversificado, en el liceo "Sistema Vivo"4. Los docentes seleccionados intencionalmente por sus competencias teórico-metodológicas reconocidas en la comunidad educativa fueron seis. La totalidad de estos docentes fueron entrevistados, la observación de clases sólo fue posible con tres de ellos, por razones de disponibilidad horaria. De cada docente, se observaron tres clases, contabilizando un total de nueve clases observadas. 
Para la producción de evidencia empírica, se desarrollaron diferentes estrategias metodológicas, teniendo presente fuentes primarias y secundarias. Entre las fuentes primarias se realizaron: entrevista semiestructurada y observación de clase no participante. Como fuentes secundarias se utilizaron materiales escritos y visuales: cuestionario a los estudiantes, protocolos y post- laboratorios, programas de química vigente para segundo y tercer año de Bachillerato Diversificado, vídeo realizado como material de apoyo para el examen práctico.

El trabajo de campo se inició en agosto de 2004, y la permanencia en el mismo de forma regular, se concretó hasta mediados de diciembre del mismo año.

\section{Entrecruzando líneas interpretativas}

El procesamiento de la evidencia empírica es un proceso en continuo progreso (Taylor; Bogdan, 1987); recorre un largo camino que comienza por las desgrabaciones de entrevistas y observaciones de clase, pasando por las audiciones, lecturas de lo que se transcribió, comentario de las clases con los docentes; más lecturas, subrayado de emergentes, búsqueda de recurrencias, dimensiones; se tienen en cuentan las anotaciones en el diario de campo, se buscan paralelismos con los documentos disponibles, y comprender los datos en el contexto en que se producen, se interpreta, se reinterpreta ... se intenta categorizar ...

La realidad del aula es compleja, la relación entre enseñanza y aprendizaje es de tipo ontológico y la intención de parcializar la complejidad obstaculiza la visión de la misma. Una mirada integradora y compleja de dicha realidad, supone una grandeza de enfoque teórico, que en el camino recorrido tan sujeto a la evidencia empírica, resultó algo lejano y, sin embargo, constituyó un desafío estimulante. Con el propósito de interpretar la evidencia empírica recogida, para comprender la realidad observada, se proponen dos dimensiones para el análisis: la reconstrucción de conocimiento en los prácticos de laboratorio y las interacciones en los prácticos de laboratorio.

A continuación, se describen algunas conceptualizaciones categóricas que cobran sentido en el contexto de la realidad observada, y por ello limitada, pero constituyendo tal vez una primera hermenéutica en estrecha relación con la empiria. Estas construcciones se realizan recorriendo las líneas que orientan esta investigación, es decir, la búsqueda de significados de los prácticos de laboratorio en la enseñanza de la química.

\section{Los prácticos de laboratorio mirados desde la reconstrucción del conocimiento}

En las diferentes actividades, que el trabajo de campo permitió compartir con los docentes, se pudo percibir en forma constante la preocupación por el conocimiento. Esta preocupación se manifestó tanto en lo personal, haciendo referencia a la formación permanente, como también en las propuestas de enseñanza, buscando optimizarlas para que favorezcan la comprensión de los estudiantes.

El conocimiento que elabora el estudiante, no es el saber erudito de la comunidad científica, tampoco es el conocimiento que el docente elaboró, sino que constituye una reconstrucción desde lo personal pero contextualizada, que depende de múltiples factores, sociales y culturales. Los docentes reconstruyen el conocimiento, y buscan diferentes caminos para potenciar dicha reconstrucción por parte de los estudiantes. En esta búsqueda, se detiene la mirada en cómo los docentes la potencian en sus alumnos, y surgen indicios al respecto: la resignificación del conocimiento cotidiano; la reformulación del error y la búsqueda de integración conceptual. 


\section{Resignificación del conocimiento cotidiano}

La relevancia del conocimiento cotidiano, constituye un hecho reconocido socialmente, así se pone de manifiesto en uno de los objetivos que plantea el programa de química para tercer año del Bachillerato Diversificado.

En las entrevistas realizadas a algunos docentes, y en los cuestionarios de los estudiantes, se percibió la importancia que para ellos reviste el conocimiento cotidiano cuando se implementan prácticos de laboratorio.

El lugar otorgado al conocimiento cotidiano, es función del rol que cada uno de los actores desempeña. Los docentes, se preocupan por la atención, el aprendizaje y la comprensión; los alumnos por recibir una enseñanza que les resulte útil para la vida. Al encontrarse en el aula estos intereses, puede decirse que están dadas las condiciones para la resignificación del conocimiento cotidiano.

La resignificación surge de la intencionalidad del docente, ya que los alumnos construyen explicaciones de la realidad en que están insertos, lejos del ámbito formal, cuando necesitan resolver situaciones prácticas, y para esto no es necesario explicitar argumentos o explicaciones, basta con actuar, si bien internamente fundamentan.

Este conocimiento construido para la acción, constituye un basamento teórico-práctico, que muy difícilmente se hace explícito. Por lo anterior, le compete al docente generar una actividad en la cual el desarrollo de la acción involucre a los alumnos, permitiéndoles verbalizar sus teorías al respecto y resignificar el conocimiento cotidiano. Tanto en entrevistas como en observaciones de clases de diferentes docentes, se encontraron elementos comunes para la resignificación: explicitación de concepciones previas y construcción social de significados y otros que pautan diferencias: preguntas generadoras y contraste - confrontación.

\section{Explicitación de concepciones previas}

Por concepciones previas, se entiende todo el conocimiento reconstruido por el alumno, tanto en el ámbito formal como informal. Estas concepciones previas están presentes siempre, el inconveniente surge cuando es necesario explicitarlas para resignificarlas desde la teoría.

La explicitación de concepciones previas, se potencia en algunos casos a través de preguntas generadoras, que conllevan a expresar la concepción previa, proveniente del saber cotidiano del alumno. Sin embargo, teniendo presente cómo se llega a la pregunta, es necesario precisar, que toda pregunta generadora surge en la interacción dialógica, como producto creativo de la intencionalidad del docente.

En diferentes instancias de diálogo entre docentes y alumnos, se observa una secuencia dialógica relacionada a lo que Mercer (1997) denomina construcción guiada del conocimiento. Para este autor, la construcción guiada del conocimiento pasa por el análisis de la utilización del lenguaje. La conversación que se genera en el aula, a partir de una cierta actividad, más allá de las diferencias de forma y contenido, se caracteriza por establecer una comprensión compartida y el lenguaje es utilizado como una herramienta y una forma social de pensamiento para el desarrollo del conocimiento y de la comprensión.

Las observaciones que los estudiantes realizan en las actividades de laboratorio se relacionan con hechos cotidianos; así la comprensión que surge de la vida diaria favorece la reconstrucción de conocimiento y el abordaje teórico del tema en cuestión. 
La explicitación de las concepciones previas de los alumnos, también es posible a través del contraste y la confrontación.

A modo de ejemplo, se puede describir cómo el docente orienta una situación de aula donde sus alumnos se enfrentan a la incertidumbre en las medidas. La incertidumbre no hace parte de una concepción de ciencia determinista ${ }^{5}$, donde se puede medir y conocer exactamente la medida. Resulta difícil para el alumno, entender que realmente nunca conoce la medida, sino que conoce el intervalo en que se encuentra, porque toda medida tiene error.

En general frente a los hechos de la realidad próxima, cualquier sujeto tiende a construir explicaciones en forma automática, en un estado particular de inconsciencia. Lo anterior se relaciona al hecho de que medir una magnitud como la longitud, resulta ser de las primeras acciones de carácter científico que cualquier individuo realiza en su vida. Cuando nos dicen que nuestra altura es de un metro y sesenta y ocho, o que nos vendieron dos metros de cinta, no surge ningún cuestionamiento sobre su veracidad pasan al ámbito de la inconsciencia dada la familiaridad con que se realizan.

La acción de contrastar y confrontar diferentes situaciones en torno del hecho de medir, despierta la concienciación sobre la relatividad de la medida. Aquí queremos apoyarnos en lo que propone Bruner (1997): el contraste y la confrontación pueden despertar conciencia sobre la relatividad del conocimiento y la metacognición -el pensamiento como objeto del propio pensamiento-, permite la elaboración de formas alternativas de concebir la creación de la realidad.

Cuando la realidad se percibe desde significados diferentes, la metacognición entra en acción aportando una base razonada para la negociación interpersonal de significados. Como el alumno interpreta desde su conocimiento cotidiano, se produce una resignificación del mismo.

\section{La construcción social de significados}

Otro aspecto a considerar, es la construcción social de significados. Ya sea por medio de una pregunta generadora o por contraste y confrontación, la respuesta que se origina en uno de los alumnos, se comparte en el contexto del grupo social, generando posibilidades de comprensión y resignificación del conocimiento cotidiano para todos los integrantes del grupo. Si bien la representación del hecho es individual, la comprensión se puede socializar.

La acción comunicativa se optimiza, porque el docente vincula la temática disciplinar con la forma en que los alumnos se expresan sobre el tema y aquí la enseñanza de la química se resignifica al proporcionarle a los alumnos nuevas formas de hablar sobre temas científicos.

La conceptualización de las diferentes temáticas abordadas en los prácticos de laboratorio, se construye en las acciones comunicativas de alumnos y docentes, y tienen en común el contexto experimental que surge como facilitador, en la resignificación de conocimiento cotidiano.

\section{La reformulación del error}

Así como en el apartado anterior, se propone "la resignificación del conocimiento cotidiano" como posible camino a recorrer para propiciar la reconstrucción de conocimiento por parte de los alumnos, aquí se plantea "la reformulación del error".

En las distintas clases observadas, surgió en la interacción dialógica del aula, el error como respuesta a los interrogantes del docente. La intencionalidad de mencionar el error, se origina en nuestra interpretación. La naturalidad con que los docentes manejan la situación, cuando

\section{8 - Universidad ORT Uruguay}


reciben respuestas incorrectas, nos habilita a decir que no son sentidos como errores. Hablamos de reformulación del error, porque cuando surge en la conversación, el docente haciendo uso de diferentes estrategias orienta el diálogo, de forma tal que cambia la estructura original de la idea en cuestión y favorece la reconstrucción de conocimiento. Los recorridos estratégicos son: el uso de analogías y la confrontación de conceptos.

\section{Uso de analogías}

La utilización de analogías es uno de los caminos que habitualmente recorren los docentes de ciencias de la naturaleza buscando construir una situación de enseñanza favorable para la comprensión de los alumnos.

En general, suelen plantearse analogías cuando los conceptos son abstractos y muy lejanos de la realidad del alumno, en diferentes situaciones de aula se pudo observar cómo los docentes utilizan la analogía como construcción estratégica tendiente a reformular el error. Ante una respuesta errónea por parte de un alumno, el docente pasa primero a recapitular los diferentes elementos que aportan en la construcción del tema, para luego retomar la pregunta y a partir de la respuesta de un alumno sustentar la analogía que permite evidenciar el error.

\section{Confrontación de conceptos}

La confrontación de conceptos la plantea el docente para poner énfasis y llamar la atención sobre la particularidad del tema abordado.

Por ejemplo en un práctico de laboratorio en el quese plantea como objetivo en la guía de trabajo: "...comprender cómo llevar a cabo medidas calorimétricas...", el docente percibe lo confuso que resulta para sus alumnos dicha propuesta, debido a la diversidad de respuestas erróneas cuando se les interroga al respecto.

En la búsqueda por reformular el error y potenciar la reconstrucción de conocimiento, el docente confronta los conceptos calor y temperatura, como una forma alternativa de organizar el campo temático y proponer una estructura que viabilice la comprensión del mismo.

\section{La búsqueda de la integración conceptual}

La actividad práctica, afirman los docentes entrevistados, debe ser sencilla, simple en su implementación, y por otro lado abarcar la multiplicidad de líneas conceptuales que se vinculan en su entorno interpretativo.

Las actividades prácticas observadas en clases que los docentes denominan demostrativas, sugieren orientar la mirada hacia la búsqueda de la integración conceptual disciplinar. En la interpretación de la realidad, surge la actividad práctica como integradora del campo conceptual en la enseñanza de la química. Se propone la búsqueda de la integración conceptual en la actividad práctica, por considerar que puede ser representativa de un tópico en particular, pero a su vez, puede ser generadora de miradas múltiples desde la teoría presentando la misma imagen. Es común la tendencia a enseñar cada concepto a través de un ejemplo o actividad práctica diferente, lo que configura ante la mirada ingenua del alumno una multiplicidad de visiones confusas y desintegradas. Para conocer tenemos que reflexionar, y el enemigo de la reflexión según Bruner (1997), es el ritmo precipitado: las mil imágenes. Con respecto al aprendizaje de las ciencias, dice que podemos tener presente lo mismo que Mies van der Rohe dijo de la arquitectura: " menos significa más".

Si bien es indiscutible, la importancia de un enfoque de enseñanza interdisciplinario, en una primera instancia la actividad práctica debe proponer la búsqueda de la integración conceptual disciplinar, para potenciar la construcción de una segunda mirada interdisciplinar. 
Es necesario precisar que no se está disgregando la enseñanza de conceptos de la de procedimientos o valores ${ }^{6}$; se quiere reivindicar la coherencia interna en la enseñanza de la química. Se espera, que el alumno construya un hilo conceptual sobre los temas trabajados en clase, a partir de la propuesta del docente, y no una gran estantería donde cada cajón contiene algo, pero permanece aislado del resto por no saber qué es lo que tienen en común. Las miradas deben orientarse desde la disciplina, para destacar los constructos representativos de significados valiosos para la reconstrucción de conocimiento.

\section{Las interacciones en los prácticos de laboratorio}

Las singularidades que reviste el universo de estudio, llevaron a considerar muy especialmente el trabajo de los docentes con las guías de laboratorio.

En un principio, llamó la atención la situación de homogeneidad que aparentemente se originaba en el hecho de que todos los docentes de la comunidad educativa, trabajaban con guías de laboratorio elaboradas por el docente coordinador. Lo anterior surge en las entrevistas, sin embargo, el entrecruce de líneas de información, permitió comprender la realidad desde otro ángulo interpretativo.

Siete de las clases observadas trabajan con guías y se desarrollan en el laboratorio. En todas ellas, se encuentran algunos elementos que denotan la diversidad de enfoques, provenientes de los estilos de enseñanza de cada uno de los docentes. Se puede mencionar, que se perfilan en todas ellas, diferentes segmentos de interacción: docente-alumnos; alumnosdocente, según la figura dominante en la escena. Los límites entre un segmento y otro se definen acordes a las secuencias de actividades previstas por el docente, los elementos que se destacan en ellos, es lo que resulta relevante en el análisis.

\section{Segmento de interacción docente-alumnos}

El segmento de interacción, docente-alumnos, puede ser identificado al comienzo de clase y al final, cuando el docente re - elabora la guía de laboratorio con los alumnos, o cuando reconstruye la actividad experimental otorgándole nuevos significados desde la teoría. En la primera fase, el docente re-elabora a través del relato la actividad experimental, haciendo hincapié en las indicaciones de la guía, y alerta sobre los riesgos y cuidados a tener en cuenta. En la segunda fase, el relato de la experiencia realizada por los alumnos, se sustenta en los modelos teóricos de representación de los fenómenos.

Las indicaciones se plantean en forma intensa en el segmento de interacciones docentealumnos, cumpliendo la finalidad de avisar al alumno sobre el contenido y lo que deben ir interpretando al respecto.

El docente planifica la actividad a realizar con sus alumnos, en este caso es la guía de laboratorio -que el alumno recibe con una semana de antecedencia-, y todo su accionar esta basado en este plan, del cual realiza el seguimiento, evalúa el alcance y comprensión que tienen los alumnos sobre el mismo.

En el seguimiento del plan surge la preocupación por corregir los errores, lo que denominamos anteriormente como reformulación.

Es importante la reconstrucción de la actividad experimental, porque a través de ella el docente pauta cuáles son los datos específicos y cómo se relacionan con los soportes teóricos que están estudiando. Así como también se hace hincapié en las conexiones directas entre lo que realizaron en la actividad y lo que el estudiante tiene que aprender, el puente que se tiende, se soporta en el conocimiento cotidiano. Con respecto a lo anterior, queremos citar a 
Lemke $(1997,28)$, “Los alumnos también necesitan detectar el contenido científico en el diálogo". Es probable dice el autor, que aprendan a practicar los juegos del aula, pero no aprendan a hablar sobre química. El diálogo científico tiene dos patrones, según Lemke, una estructura de actividad y un patrón temático.

El docente y los alumnos, se involucran estratégicamente, en un juego de expectativas en cuanto a lo que puede suceder, la estructura de actividad. A través de los datos el docente busca la interpretación del fenómeno desde la teoría, en un juego de preguntas, ejemplos, cambios sugestivos de voz, comparaciones y enunciados, constituyendo así una estructura de actividad.

En forma simultánea con lo anterior, se elaboran significados en torno a los procesos involucrados, utilizando términos y símbolos relacionados con el tema, lo que constituye un patrón temático. Los alumnos saben a qué hace referencia la terminología en cuestión, pero no son capaces de explicar cómo se relacionan sus significados. Para hacerlo y poder hablar científicamente, necesitan conocer el patrón de vinculaciones de significado, el patrón temático.

\section{Segmento de interacción alumnos-docente}

Este segmento de actividad se caracteriza por el trabajo en grupo de los alumnos durante la experimentación, el docente acompaña la actividad. Cada grupo, p.e. sexto medicina se subdivide en tres subgrupos para el curso práctico, la observación se realiza siempre en el mismo subgrupo. El subgrupo de práctico tiene aproximadamente diez alumnos y para la experimentación se subdividen en dos grupos, esto se debe al limitante del material con que cuenta el laboratorio.

El trabajo en grupo, representa una modalidad de trabajo muy valiosa para la formación de los estudiantes. Los prácticos de laboratorio favorecen esta modalidad, y con ella la toma la de decisiones; la implicación, la cooperación, la discusión y argumentación de ideas y un proceso secuencial de integración de las diversidades comportamentales de cada individuo. Los valores atraviesan esta situación: tienen que ser solidarios, escuchar al otro, respetar las diversas opiniones, negociar la toma de decisiones, etc.

En este segmento de interacción el alumno es el que interroga, cuestiona y el docente pasa a tener una actitud más pasiva, a orientar el diálogo, invitando a la participación los alienta a construir la idea que están desarrollando, comprometiéndolos a continuar en la actividad. El énfasis está en ayudar al alumno, proporcionando las condicionantes útiles para que ellos desarrollen las estrategias que entiendan convenientes. Aquí el docente se perfila como potenciador en el desarrollo de los estudiantes como personas autónomas, a través de experiencias educativas de significación personal.

En el relato de los docentes entrevistados se evidenció preocupación por dar participación directa al alumno en la actividad experimental, para que él pueda sentirse el actor principal en la toma de decisiones, desde el rol de experimentador. La finalidad del docente en la actividad experimental, está orientada a preparar al estudiante para elegir, elaborar y evaluar lo que aprende.

En los segmentos de interacción alumnos-docente, se generan espacios de participación que permiten al alumno involucrarse libremente y el docente sólo guía, sugiere, alienta, propicia y previene. En este sentido el alumno se siente partícipe real de la situación. En los cuestionarios realizados a los alumnos, surgió unánimemente la preferencia por las clases de química que se desarrollan con prácticos de laboratorio. 
Los alumnos perciben los prácticos de laboratorio como actividades que los involucran, que sustentan canales de comunicación favorables y les ayudan en la comprensión., aquí surge con mucha fuerza la siguiente expresión, “... La comprensión se presenta cuando la gente puede pensar y actuar con flexibilidad a partir de lo que sabe” (Perkins, 1999, 72).

\section{Una interacción mediada: la puesta en escena}

Aquí se hace referencia a un vídeo en el que se relatan los prácticos de laboratorio que constituyen el curso práctico. El mismo, hace parte de los documentos analizados, consiste en un videograma, es decir un mensaje grabado en soporte magnético obtenido a través de una videocámara. Este registro audiovisual aborda los prácticos de laboratorio de química, de quinto y sexto año-segundo y tercer año del Bachillerato Diversificado-, y es elaborado por un grupo de docentes con la colaboración de alumnos.

Las prácticas allí registradas, hacen parte del temario de examen práctico de cada uno de los cursos mencionados anteriormente y tiene como finalidad apoyar al alumno en la preparación del mismo.

Consiste en el relato de las guías de práctico, acompañado de la acción llevada a cabo por una alumna protagonista. En forma sincrónica, se desarrolla el mensaje y se realiza el procedimiento del práctico y la explicación de lo observado. Esta grabación se puede entender como una interacción mediada por el recurso audiovisual, acompañada de elementos dinamizadores como el color, la imagen y la música. La calidad de imagen y sonido de la grabación es buena, permite una audición clara y una visión nítida de los cambios químicos. Existe una secuenciación entre imagen fija e imagen en movimiento. Es decir, alguien realiza el relato y describe lo que se está haciendo en el procedimiento. La imagen en movimiento muestra la manipulación, la coloración de los reactivos, los cambios de color cuando se obtiene un precipitado y, la explicación sobre los cambios observados se realiza con imagen fija sobre visor de computadora. Allí van apareciendo las fórmulas de las especies químicas que se mencionan, y en el caso de cambio de color las fórmulas se inscriben en recuadros del color de referencia. La secuenciación de imágenes fija y en movimiento es acompañada por intervalos musicales.

En términos generales, el registro de vídeo se puede describir como totalizador y dinámico. Esto último se argumenta en la ágil combinación de elementos: imagen en movimiento, relato, música, imagen fija, etc. Lo de totalizador lo sugerimos a nivel del lenguaje, entendiendo que el mensaje narrado se efectiviza mediante una forma del mismo ya que abarca todo el espacio perceptivo de modo simultáneo y total.

Si se focaliza la atención en el mensaje que se quiere comunicar, esto lleva a pensar en las funciones epistemológicas de los relatos y en el aporte de Jackson $(2002,28)$, cuando dice “...En muchos casos los relatos no sólo contienen un saber, sino que son en sí mismos el saber que queremos que los estudiantes posean".

Lo anterior, sugiere que el mensaje del vídeo dirigido a los estudiantes para que les sirva de material de apoyo, constituye una estructura innovadora en el planteo del lenguaje experimental en la enseñanza de la química. En el lenguaje experimental, se relacionan los conceptos que permiten comprender los diferentes fenómenos observables. El recurso audiovisual potencia la comprensión de este lenguaje, porque permite centrar la atención sobre lo que realmente importa, en menos tiempo y en forma dinámica.

La comprensión que el alumno debe hacer sobre lo que observa o experimenta en un práctico de laboratorio, en la grabación aparece totalizada, es decir, no ocurre como en el curso, que primero realiza el experimento y después en el post-laboratorio se explica lo observado. 
Retomamos lo referente a las funciones epistemológicas de los relatos, en el entendido de que cada práctico de laboratorio que forma parte de este documento visual, constituye una estructura de conocimiento que potencia la comprensión del estudiante y podría resignificar la enseñanza de la química, si se proponen nuevos caminos para transitar.

\section{Reflexiones finales}

Los prácticos de laboratorio representan una figura destacada en las prácticas de enseñanza de los docentes investigados, más allá de que para ellos se presente como una prescripción curricular. A nivel del Bachillerato Diversificado, lo prácticos de laboratorio hacen parte de la propuesta programática, allí se indican cuáles y cuándo se deben realizar para cumplir con el desarrollo del curso práctico.

La propuesta curricular genera un divorcio entre teoría y práctica, originando una ambivalencia que se percibe, tanto en el discurso de los docentes como de los alumnos. En la totalidad de los cuestionarios de los alumnos, se registró que la actividad práctica se realiza para aplicar lo visto en el curso teórico. Los docentes argumentan las siguientes finalidades para la realización de actividades prácticas: motivación, aplicación o demostración de algún principio, recolección de datos para comprobar algo. También mencionan que para ellos las actividades prácticas más interesantes son aquellas que sorprenden al alumno, que despiertan la curiosidad. Los docentes seducen a los alumnos a través del discurso y la actividad práctica propuesta.

El conocimiento práctico del docente, que sustenta todas sus decisiones de enseñanza, no siempre llega al plano de la verbalización, por eso tal vez surgen las aparentes contradicciones cuando hablan de guías que se aplicarían como recetas, pero que no condicen con lo observado. Es decir, la demanda de la propia enseñanza por ser una actividad práctica, hace que el docente actualice su conocimiento práctico, que es implícito, y cuyos enunciados formales aún no se han concretado. Todo este proceso se enriquecería en situaciones de intercambio y socialización de las diferentes propuestas de enseñanza. Las horas de coordinación que el currículo propone a nivel de la enseñanza media obligatoria, no se continúan para la etapa post-obligatoria, en este caso el Bachillerato Diversificado. Dado que los recursos salariales son menguados y reducidos, tendrían que generarse estrategias institucionales que permitieran la coordinación. El intercambio de ideas y espacios para evaluar los materiales curriculares como por ejemplo las guías de laboratorio, aún no se han conquistado. En los comentarios realizados por los docentes, parecen surgir desacuerdos en cuanto a la viabilidad del uso de guías, pero igualmente se siguen implementando, tal vez por falta de tiempo para la discusión y elaboración de nuevas propuestas.

La enseñanza es una actividad práctica. La enseñanza de la química se reviste de singularidades que hacen a como se construye el conocimiento disciplinar. Un alto porcentaje del conocimiento se construye mediante la experimentación en los laboratorios. Lo anterior suele llevar a confusiones, ya que suele percibirse la enseñanza de la química, por un camino semejante al recorrido en la construcción de la disciplina.

En las clases observadas, los prácticos de laboratorio se realizan a partir del material escrito preparado con antecedencia. Si bien el alumno posee una semana antes la guía de laboratorio, los docentes observados reelaboran la guía junto con sus alumnos, o brindan un espacio de lectura e intercambio entre los alumnos antes de realizar la actividad. En ninguno de los casos la guía se convierte en una receta, ya que en todo momento se cuestiona al alumno en torno a lo que se hace. 
En nuestro universo de estudio, prima la homogeneidad en relación con las guías, si bien se evidencia en las clases que la actividad práctica, más allá de seguir una guía de trabajo, se construye con características singulares, como producto de la interacción del docente con su estilo de enseñanza y del respectivo grupo social.

Los prácticos de laboratorio, mirados desde la reconstrucción del conocimiento, sugieren la resignificación del conocimiento cotidiano, la reformulación del error y la búsqueda de la integración conceptual. El docente desarrolla actividades que involucran al alumno permitiéndole explicitar sus conocimientos teóricos prácticos de la realidad inmediata. La resignificación del conocimiento cotidiano, se observó en dos niveles: a través de la explicitación de concepciones previas, tanto por preguntas generadoras como por contraste y confrontación, y en la construcción social de significados en la que la representación de los hechos es individual pero la comprensión de los mismos se puede socializar.

Si bien se eligió el término error, es necesario realizar una precisión: en el aula de los docentes observados, el error no se identifica como tal. El clima del aula siempre se observó agradable y los alumnos se expresan libremente. Lo que desde la disciplina representa un equívoco, el docente no lo manifiesta como tal, sino que lo retoma y en una secuencia de preguntas e intercambios de ideas hace que los alumnos construyan la respuesta acorde a la situación. La reformulación del error se realiza a través del uso de analogías o confrontación de conceptos.

La búsqueda de la integración conceptual sugerida, se da en torno a las actividades prácticas. Estas actividades constituyen un peldaño previo a las actividades interdisciplinarias. Es decir, es conveniente pasar de la enseñanza fragmentada de la disciplina a una propuesta integradora de la misma, esto significa que podría ser abarcadora de varios tópicos en el contexto de representación correspondiente.

Las interacciones en los prácticos de laboratorio constituyen una dimensión para el análisis, desde la cual surgen: los segmentos de interacción docente-alumnos y alumnos-docente, y una interacción mediada: la puesta en escena.

En las clases observadas, donde se trabajó con material de antecedencia, ya sea con las guías de práctico o con las guías de post-laboratorio, se observan segmentos de interacción, según la figura predominante en el diálogo docente-alumnos o alumnos-docente. En el segmento de interacción docente-alumnos encontramos dos instancias: en una, prevalecen las orientaciones dadas por el docente y en la otra, la reconstrucción de la actividad desde la teoría. En el segmento de interacción alumnos-docente, el papel principal le corresponde al alumno, participando libremente de la actividad y al docente le corresponde guiar, sugerir, alentar, propiciar y prevenir. Aquí es necesario hacer una acotación: si bien tenemos condiciones de describir lo que observamos en los segmentos mencionados anteriormente, no podemos decir lo mismo de un tercer segmento alumno-alumno, que si bien lo percibimos, no recogimos evidencia empírica para describirlo. Este segmento se inserta en los anteriores como resultado de los espacios en que los alumnos trabajan activamente.

Aquí se abren algunos interrogantes y se suman a los de partida. En los datos recogidos, podríamos evidenciar, que el énfasis en los procesos cognitivos está en la observación y formulación de hipótesis. Lo anterior se percibe claramente en las clases donde no se trabaja con guías, pero en las que sí se hace, nos surgen interrogantes con respecto al alumno: ¿formula hipótesis espontáneamente, observa lo que solicita el docente? ¿la interacción con los pares, qué aporta?

\section{4 - Universidad ORT Uruguay}


Si bien no pretendíamos observar a los alumnos, esto resultó un obstáculo; nos encontramos con que no es posible dar respuestas con relación a los procesos cognitivos que se privilegian en los prácticos de laboratorio. Lo observado nos sugiere, que los docentes estimulan los procesos cognitivos; buscan el encadenamiento de ideas; insertan la modelización cuando es necesario; favorecen las decisiones interactivas; pero no pueden concretar al respecto sin información proveniente de los alumnos.

A partir de la evidencia empírica surgen indicios, en los que el desenvolvimiento de estrategias cognitivas por parte del alumno, se abordan desde los niveles del lenguaje que este utiliza para comunicar, discutir y argumentar sus resultados, así como también el nivel de análisis que realiza, desde la observación de detalles pequeños, hasta situaciones hipotéticas de aplicación y resolución.

Uno de los docentes realizó actividades prácticas, fuera del contexto de aula, a partir del interés manifestado por los alumnos. Si bien ya se habían realizado cuando ingresamos al campo, en los cuestionarios de los alumnos surgen como actividades destacadas. Estas actividades que se realizaron sin guías, voluntariamente, y no hacen parte de la evaluación del curso, son las que los alumnos recuerdan. Lo anterior hace que nos cuestionemos sobre el trabajo con guías de laboratorio: ¿su implementación abre realmente puertas a la curiosidad y creatividad de los alumnos?

En la mirada dirigida a las interacciones en las actividades prácticas, entendemos conveniente rescatar y resignificar, desde la teoría, un dato singular: una interacción mediada: la puesta en escena de los prácticos de laboratorio. Si bien en su contexto, representa un material curricular de apoyo a situaciones de evaluación, queremos rescatarlo más allá de esa función. La puesta en escena representa un recurso audiovisual, en el que la relación teoría-práctica no se presenta disociada o como una al servicio de la otra, sino que se han reconciliado. En la comunidad educativa donde hemos investigado, presenciamos muchos esfuerzos insulares; tenemos la impresión de que mucho de lo que observamos en nuestra estadía en el campo pasa por un estado de inconsciencia del colectivo, en el cual materiales curriculares como la puesta en escena, que destacamos como un potenciador para la resignificación de la enseñanza de la química, permanece en el anonimato y no se utiliza en el aula.

A modo de cierre queremos reflexionar sobre algunos puntos que encontramos relevantes durante este proceso de investigación vivido muy intensamente. Las actividades prácticas o prácticos de laboratorio, tendrían que comenzar a descontextualizarse de la situación en la que hoy adquieren sentido propio; es decir, surge la necesidad sentida desde la relación teoríapráctica de buscar nuevos contextos para la realización de las mismas.

Los prácticos de laboratorio aportan a la reconstrucción de conocimiento en la enseñanza de la química, pero el contexto ambivalente de la relación teoría-práctica, requiere una reflexión urgente sobre las tendencias tecnicistas que se prescriben desde el currículo anulando la autonomía del docente.

La labor educativa en todas sus dimensiones se presenta complejizada, la imposición de enfoques, metodologías o estrategias didácticas, a través del currículo, fragilizan y desacreditan aún más el rol del docente como profesional de la enseñanza, por lo cual entendemos necesario rescatar y poner énfasis en algunos elementos que no se están teniendo en cuenta desde una óptica globalizadora: lo pedagógico, lo moral, lo ético y lo político. 


\section{Bibliografía y notas}

Adúriz Bravo, A. 2000. Elementos de teorías y de campo para la construcción de un análisis epistemológico de la didáctica de las ciencias. En: Enseñanza de las ciencias 18(2): pp. 347-378.

Bruner, J. 1997. La educación puerta de la cultura. Madrid: Visor.

Bunge, M. 1976. La investigación científica. Barcelona: Ariel.

Caamaño, A. 2003. Los trabajos prácticos en ciencias. En: Jiménez, A. Enseñar ciencias. Barcelona: Graó. pp. 95-118.

Camilloni, A. 1997.De herencias deudas y legados. En: Camilloni A.; et al.. Corrientes didácticas contemporáneas. Buenos Aires: Paidós. pp. 17-40.

Cañal, P. 1987. Un enfoque curricular basado en la investigación. En:. Investigación en la escuela 1: pp. 43-50.

Carmen, L. 2000. El Análisis y secuenciación de los contenidos educativos. Barcelona: Horsori.

Corominas, J. ; Lozano, T. 1994. Trabajos Prácticos para la construcción de conceptos: experiencias y experimentos ilustrativos. En: Alambique, 2: pp. 21-26.

Díaz Barriga, Á. 1998. Didáctica y currículum. México: Paidós.

Fenstermarcher, G. 1989.Tres aspectos de la filosofía de la investigación sobre la enseñanza. En: Wittrock, M. La investigación de la enseñanza I. Madrid: Paidós. pp. 150179.

Gimeno, J. ; Pérez, A. 1995. Comprender y transformar la enseñanza.4ta.ed. Madrid: Morata.

Grau, R. 1994. ¿Qué es lo que hace difícil una investigación? En: Alambique 2:pp. 27-35.

Insaustí, M. 1997. Análisis de los trabajos prácticos de química general en un primer curso de universidad. En: Enseñanza de las Ciencias 15(1): pp. 123-130.

Jackson, P. 2002. Práctica de la enseñanza. Buenos Aires: Amorrortu.

Lemke, J. 1997. Aprender a hablar ciencia. Barcelona: Paidós.

Litwin, E.1997. Las configuraciones didácticas. Buenos Aires: Paidós.

Mercer, N. 1997. La construcción guiada del conocimiento. Barcelona: Paidós.

Perkins, D. 1999¿Qué es la comprensión? En: Stone Wiske, M. (comp). La enseñanza para la comprensión. Buenos Aires: Paidós. pp. 69-92.

Pozo, I. 1996. Aprendices y maestros. Madrid: Alianza.

Rodríguez Moneo, M.1999. Conocimiento previo y cambio conceptual. Buenos Aires: Aique.

Stodolsky, S. 1991. La importancia del contenido en la enseñanza. Buenos Aires: Paidós.

Taylor, S; Bogdan, R. 1987. Introducción a los métodos cualitativos de investigación. Barcelona: Paidós.

86 - Universidad ORT Uruguay 
${ }^{1}$ En nuestro país, se encuentran vigentes tres planes de estudio para la enseñanza media superior o post-obligatoria, el de más larga data es el Bachillerato Diversificado al que hacemos mención.

${ }^{2}$ Las guías de laboratorio también se reconocen bajo la denominación de protocolos o técnicas.

${ }^{3}$ La didáctica es una disciplina que surgió en el siglo XVII para abordar los problemas de la enseñanza en la relación maestro - alumno. Un elemento que signa su elaboración es la ausencia de una dimensión institucional referida al sistema educativo, mientras que la concepción curricular obedece a las exigencias de una educación obligatoria, que pauta la conformación de los sistemas educativos, en el contexto que exige la industrialización monopólica. Díaz Barriga, 1998.

${ }^{4}$ La institución educativa como sistema, es abierto; son diversos y complejos los ámbitos en que interactúa relacionándose con el medio. La realidad percibida por sus actores, no siempre reviste las mismas características. Las diferentes concepciones se entrecruzan e intercambian significados enriqueciendo y complejizando las funciones vitales que allí se cumplen. No encontramos otra forma para expresar lo que interpretamos: la institución donde investigamos es "Un Sistema Vivo". Esta denominación de la institución, no pasa sólo por una búsqueda de anonimato, sino que por el contrario, es un logro construido desde nuestra percepción de la realidad, desde el momento en que optamos por recorrer la institución por la ruta de la investigación educativa.

${ }^{5}$ Esta concepción de ciencia se percibió en la totalidad de los cuestionarios aplicados a los alumnos.

6 "Las estrategias, descritas en términos de competencias, aisladas de los contenidos, reflejan una concepción superada que reduce la enseñanza a habilidades del pensamiento, y que separa la enseñanza de los conceptos de la de los procedimientos y valores. " (Litwin,1997,..41)

*El Artículo se enmarca en la tesis de maestría de la autora, dirigida por la Mag. María Teresa Cafferata. Año 2005.

**Master en Educación, Universidad ORT Uruguay. Diploma en Educación, Universidad ORT Uruguay. Profesora de Educación Media, Instituto de Profesores Artigas. Docente, Centro regional de Profesores del Norte. Docente, Consejo de Enseñanza Secundaria. 